\title{
Determining the full halo coronal mass ejection characteristics
}

\author{
V. G. Fainshtein \\ Institute of solar-terrestrial physics SB RAS, \\ P.O. Box 291, 664033, Irkutsk, Russia \\ email: vfain@iszf.irk.ru
}

\begin{abstract}
In this paper we determined the parameters of 45 full halo coronal mass ejections (HCMEs) for various modifications of their cone forms ("ice cream cone models"). We show that the CME determined characteristics depend significantly on the CME chosen form. We show that, regardless of the CME chosen form, the trajectory of practically all the considered HCMEs deviate from the radial direction to the Sun-to-Earth axis at the initial stage of their movement.
\end{abstract}

\section{Introduction}

The full halo CME (HCME) was first reported by Howard et al. (1982). A lot of HCMEs were detected by LASCO [Yashiro et al. (2004)]. Full HCMEs are considered to move from the Sun to the Earth if they are accompanied by activity on the visible disk of the Sun [Webb et al. (2000)]. Full HCMEs are responsible for many large geomagnetic storms [Webb et al. (2000)]. To time the arrival of such CMEs to the Earth and predictions their geoeffective parameters at $R=1 A E$ it is necessary to determine the true full HCME characteristics near the Sun. In the papers [Zhao et al. (2002); Michalek et al. (2003); Hie et al. (2004); Hue et al (2005); Michalek (2006) and Fainshtein (2006)] some techniques of finding the true full HCME parameters in 3-D space were proposed. In the most techniques CMEs are supposed to have a cone form [Howard et al. (1982); Fisher \& Munro (1984)]. But, as observations of limb CME show, the form of every CME is best approximated by only one of the cone form three modifications ("ice cream cone models", Fisher \& Munro (1984)). In this paper parameters of 45 full HCME for three possible modifications of their cone form are determined using the method [Fainshtein (2006)]. We show that these parameters essentially depend on the CME form chosen modification.

\section{Determining the observed full halo CME characteristics}

Our analysis showed that there is a positive correlation between the eruptive prominence and/or the limb post-eruptive arcade angular size $\delta_{P-A}$ and the $2 \alpha$ angular size of the LASCO C3 CME related to the prominence (arcade), [Fainshtein (2006); Fainshtein (2007)]. The regression line equation for this correlation is $2 \alpha=-0.18 \delta_{P-A}{ }^{2}+10.16 \delta_{P-A}$ +11.3 .

According to Fainshtein (2006), we will assume that this regression line also relates the eruptive filament (EF) angular sizes and/or the post-eruptive arcade (PEA) on the visible disk of the Sun to the EP (PEA) - related full HCME angular size. Then, to find angular size of such CMEs one may use the above regression line, in which $\delta_{P-A}$ will mark now the EF (or PEA) angular size on the visible disk of the Sun. To determine other parameters of the full HCMEs we used the relations between the halo CME characteristics obtained within the CME cone model three modifications, Fig. 1. For these models in this 


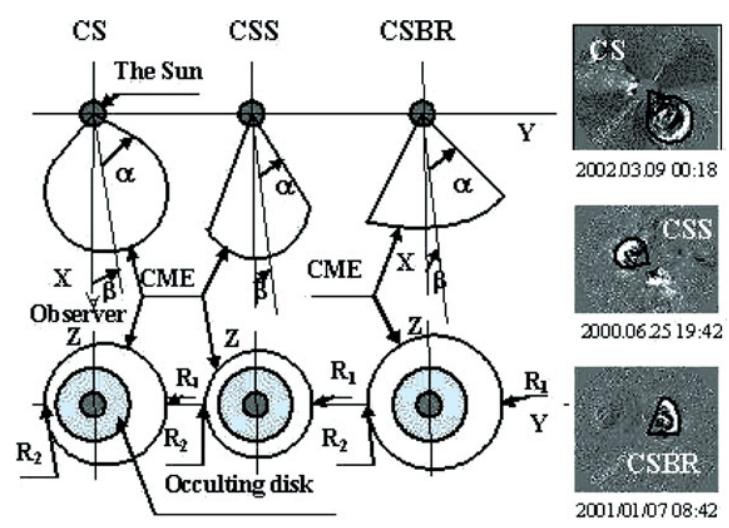

Figure 1. The coronal mass ejection models. A1-C1: the image of the CME moving at $\beta$-angle to the Sun-to-Earth axis (X-axis) in the plane $\mathrm{X}-\mathrm{CME}$ axes. The $\mathrm{Y}$-axis is perpendicular to the $\mathrm{X}$-axis and is located in the plane of the $\mathrm{X}$-axis - CME axes as well as in the plane of the sky. A2-C2: outer-boundary images of a model halo CME in the plane of the sky (plane YZ). To the right of the CME models are the examples of the limb CMEs whose outer boundary form is close to each of model forms.

paper we will use the following designations: CS , CSS, and CSBR models. These models are different in the relation between the cone base size and the size of the structure on which the cone leans. To simplify, we assume the cone base form is a circle, and the cone leans on the part of the sphere in this paper. Using Fig. 1, we may obtain the expressions relating the full HCME angular size with its other parameters. To illustrate this we will give two formulas for the CS model:

$$
\begin{gathered}
\sin \beta=\frac{R_{1}-R_{2}}{R_{1}+R_{2}} \sin \alpha \\
V_{F E}=V_{1} \frac{\cos \beta+\sqrt{\sin \alpha^{2}-\sin \beta^{2}}}{\sin \alpha+\sin \beta}
\end{gathered}
$$

Here, $\beta$ is the angle between HCME axis and the Sun - to - Earth axis, $V_{1}$ is the velocity of a point with radius $R_{1}, R_{1}$ is the largest, and $R_{2}$, respectively, is the smallest positions of the HCME image boundary in the plane of the sky, $V_{F E}$ is the CME front velocity along the Sun-to-Earth axis.

To test the given method of determining the full HCME parameters, 45 coronal mass ejections associated with the eruption of filament (EF) and/or post-eruptive arcade (PEA) have been selected. The EF and PEA characteristics were determined by Sun images in the FeXII $\lambda$ 195A extreme ultraviolet line (SOHO/EIT). The procedure of the EF (PEA) angle size and values $R_{1}$ and $R_{2}$ determination is described in the paper Fainshtein (2006). For analysis we also used the $\beta_{P-A}$ angle position of the EF and/or EPA center (within the heliocentric coordinate system). As V1 values we used the linear fit velocities from the "LASCO CME catalog" (http ://cdaw.gsfc.nasa.gov/CME_list/).

\section{Results}

(i) It is evident from the our analysis that the CME parameters revealed by using various models differ distinctly. For example, $<\beta>(C S$ model $)=10.3^{\circ} ;<$ $\beta>(C S S$ model $)=15.4^{\circ} ;<\beta>(C S B R$ model $)=17.05^{\circ}$, and $<V_{F E} / V_{1}>$ 


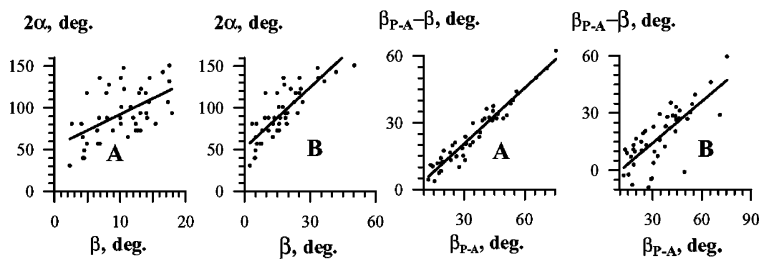

Figure 2. The $\beta_{P-A}$-angle dependence of the CME $2 \alpha$ angular size and the $\beta_{P-A}-\beta$ correlation with the $\beta_{P-A}$ value. $\mathrm{A}$ is the CS model, $\mathrm{B}$ is the CSBR model.

$(C S$ model $)=2.3 ;<V_{F E} / V_{1}>(C S S$ model $)=2.05 ;<V_{F E} / V_{1}>(C S B R$ model $)$ $=1.4$. These differences should be considered significant if one applies, e. g., the obtained results to determine the halo CME transit time between the Sun and Earth. Therefore, one has to justify the usage of this or that model in each particular case.

(ii) The $\beta$-angle differs significantly from the $\beta_{P-A}$ angular position of the eruptive filament (of the post-eruptive arcade)related to the HCME. The author believes that this inequality reflects halo CME trajectory deviation from the radial direction towards the Sun-to-Earth axis at the initial stage of their movement. The physical mechanism of such a CME trajectory peculiarity is proposed in the paper Fainshtein (2007).

(iii) With the $\beta_{P-A}$ (the value of angle position of the CME-related eruptive filament (post-eruptive arcade)) increase, the value of the $\left(R_{1}-R_{2}\right) /\left(R_{1}+R_{2}\right)$ parameter, that characterizes the halo CME center shift in the plane of the sky relative to the solar disk center along the HCME large axis, also increases.

(iiii) The bulk of the considered full halo CMEs have relatively large angle size with the mean value of $93^{\circ}$. This value is essentially larger than the "limb" CME mean angular size equal to $\approx 45^{\circ}$ [Yashiro et al. (2004). The similar conclusion was made earlier in the papers [Michalek et al. (2003); Fainshtein (2006)]. The CMEs, whose axis are most deviated from the Sun-to-Earth axis, have, on average, larger angle size that the CMEs moving near the Sun-to-Earth axis. In its turn, the $\beta-\beta_{P-A}$ angular difference increases with increasing $\beta_{P-A}$, on average (Fig. 2).

Acknowledgements. SOHO is a project of international cooperation between ESA and NASA. This work was supported by RF Leading Scientific Schools Support Governmental Grant SS 4741.2006.2 and by P-16 RAS Presidium Fundamental Research Program.

\section{References}

Fainshtein, V. G. 2006, Geomagnetism and aeronomy, 46, 384

Fainshtein, V. G. 2007, Cosmic Research, 45, 384

Fisher, R. R. \& Munro, R. H. 1984, Astrophys. J., 280, 428

Howard, R. A., Michels, D. J., Sheeley, N. R. Jr., \& Koomen, M. J. 1982, Astrophys. J., 263, L101.

Michalek, G., Gopolswamy, N., \& Yashiro, S. 2003, Astrophys. J., 584, 472

Michalek, G. 2006, Solar Phys., 237, 101

Webb, D. F., Cliver, E. W., Crooker, N. U., St. Cyr, O. C., \& Thomson, B. J. 2000, J. Geophys. Res., 105, 7491

Xie, H., Offman, L., \& Lawrence, G. 2004, J. Geophys. Res., 109, A03109, doi:10.1029/ 2003JA010226

Hue, X. H., Wang, C. B., \& Dou, X. K. 2005, J. Geophys. Res., 110, A08103, doi:10.1029/ 2004A010698

Yashiro, S., Gopalswamy, N., Michalek, G., St. Cyr, O. C., Plunkett, S. P., Rich, N. B., \& Howard, R. A. 2004 J. Geophys. Res. 109, A07105

Zhao, X. P., Plunkett, S. P., \& Liu, W. J. 2002, J. Geophys. Res., 107, SSH (13-1), doi10.1029/2001JA009143 